\title{
A principled approach to the design of collaborative MOOC curricula
}

\author{
Stian Håklev ${ }^{1}$ and James D. Slotta ${ }^{2}$ \\ 1 Ecole Polytechnique Fédérale de Lausanne, 1015 Lausanne, Switzerland \\ stian.haklev@epfl.ch \\ 2 Boston College, 140 Commonwealth Ave. Chestnut Hill, MA 02467, USA \\ slotta@bc.edu
}

\begin{abstract}
MOOCs have the potential to benefit from the large number of very diverse learners that participate in courses, but this requires a principled approach to MOOC curriculum development. Courses need to take into consideration the diversity of learner experience and intentions, and incorporate scripts that both benefit from the large numbers of learners (crowd-sourcing), as well as enabling small-scale intense collaboration. The real challenge is tying together a set of learning activities and the development of a community knowledge base, with the specific curriculum learning goals of the course. This paper offers a pragmatic approach to developing courses, based on the experience of a MOOC for teacher professional development.
\end{abstract}

Keywords: MOOCs, scripting, collaborative learning

\section{Introduction}

In a few short years, MOOCs have grown from a niche phenomenon, to a large scale movement involving universities, companies and organisations around the world. Thousands or tens of thousands of learners participate in courses, with very different backgrounds, levels of existing knowledge and motivation, and goals for their learning. This large number and distributed nature of participants in MOOCs suggests opportunities for innovative pedagogical designs, however the realities to date typically place the learner in an isolated context, with little direct peer interaction or sense of participation in a collective enterprise.

Researchers have already begun pushing at the boundaries of technology and pedagogy. Some have focused on the peer assessment, such as Dan Hickey, who launched a Big Open Online Course (BOOC), building on his research on peer assessment and WikiFolios for collaborative learning, where students engaged in small groups to promote and reflect upon the works of their peers [5].

Others have experimented with building better peer review systems embedded into traditional MOOC platforms, such as the PeerStudio platform [6]. There have also been attempts at designing stand-alone social platforms that are connected with the traditional MOOC platforms, such as the ProSolo system from the Data, Analytics and Learning MOOC (DALMOOC) [2]. 
To strengthen the development of collaborative courses, there is a need for theoretically informed design principles and frameworks that integrate curriculum development, pedagogical scripting, and technological supports. This paper will present a pragmatic model for developing complex collaborative learning scripts that cater to, and benefits from a diverse student population, and responds to curriculum learning goals.

\section{A Knowledge Community approach to MOOC design}

One of the fundamental tenets of Computer Supported Collaborative Learning is the idea that interaction with other learners can improve learning outcomes. Students are forced to externalize their ideas, working with multiple shared representations of knowledge structures, and collaborating to converge on a shared understanding of meaning [3].

In addition, learning theories such as Connectivism [1] and social constructivism [7] emphasise the added value of diverse student viewpoints, experiences and identities to the learning experience. To increase the chance of real life transfer of learning, and applicability of theory to practice, it is necessary for learners to connect what they are learning with their own lives, and those of others. Especially in social science topics, the experiences and ideas from other students can be very valuable contributions to the learning process.

These learning theories become increasingly relevant given the emergence of user-created media, and the radical shift in how we access information and learn about the world. We learn by formulating and expressing our ideas and theories in language, posting on discussion forums and tweets. We gain access to deeply personal perspectives of others through personal blogs. We build on each other's knowledge by negotiating the successive editing of Wikipedia articles. And we harness input from thousands of peers through aggregation mechanisms like voting, folksonomic tagging, and even automated classification.

Knowledge Community and Inquiry (KCI), is a pragmatic framework for curriculum development to foster knowledge communities, which advocates scripting and coordinated grouping to assure comprehensive distribution across a targeted domain, but adds a layer of collective knowledge building, where students engage with Web 2.0 technologies to develop a shared knowledge base that serves as a resource for their subsequent inquiry [9].

Making ideas visible and accessible is at the heart of learning in a KCI design. This requires the use of existing Web 2.0 technologies, sometimes augmented by bespoke technology platforms or supports, as well as a pedagogical designs. Students work individually or in small groups collecting information, brainstorming ideas or solutions, supported by carefully designed prompts and scaffolds. Small group work alternates between cooperative work - divide and conquer - and collaborative - where group members build upon each other's knowledge.

Many KCI designs feature a group project in which students collaborate throughout the term, with new elements or dimensions added as the students gain access to a larger individual and community knowledge base, and become 
more conceptually sophisticated [10]. Such persistent, revisited projects can function as a means of guiding students' connection of principles throughout the course. Further, given that the projects are indexed to the learning objects of the course, these inquiry projects can support the summative evaluation of student learning.

\section{Adapting the KCI model to MOOCs}

A number of design studies have tested the KCI model in primary and secondary classrooms, and university courses. In adapting the model for MOOCs, we faced a number of specific challenges, which will be addressed below. Students are much more diverse in terms of their location, experience, age, level of motivation, and what they want out of the course. The number of learners is much higher, making traditional social activities difficult (a discussion with 2,000 people becomes a cacaphony), and the largely asynchronous nature of MOOCs makes social coordination and a sense of social presence difficult to achieve.

The MOOC featured in this study was designed to support in-service teachers to integrate inquiry and technology into their lessons. It was explicitly marketed to in-service teachers, and was designed to build upon their professional experience and respond to their real challenges, providing tools, examples and approaches that could be directly applied within their professional settings. The course came out of a collaboration between the University of Toronto Schools (UTS), a university-affiliated private secondary school, and the Encore research group at University of Toronto led by Dr. Slotta, enabling us to provide an integration of academic and theoretical ideas, with applied practice.

The course ran on the EdX platform, however we designed several supplementary learning activities, which ran on a server hosted at the University of Toronto, and which were embedded within EdX pages using the Learning Tools Integration (LTI) protocol [8]. Below we will outline a number of the design features of the MOOC.

\subsection{Special Interest Groups}

Given that the course was catering to a wide variety of teachers, we decided to divide students into Special Interest Groups. Students went through a questionnaire on the external LTI site identifying their teaching level, subject area, and providing folksonomic tags for their specific interests. In order to have the course design be informed by its future learners, we opened a "pre-course lounge", where teachers could register, fill out the questionnaire, and begin submitting useful resources-all several weeks before the actual course began running.

Taking this initial data into consideration, we generated an initial list of Special Interest Groups, designed to group logically similar teaching levels and subject areas. The ability to gauge student demographics and interests before the course began, proved very important when it comes to the Higher Education and Online Learning SIG. Initially, we had targeted the course specifically towards 
K-12 teachers, and not planned to include any groups for non-K-12 students, however upon seeing the initial interest, we were able to adjust our plans, and the Higher Ed SIG in fact turned out to be the most popular one.

The SIGs were generated on our external server, however we imported the CSV export into EdX several times per day to generate cohorts based on SIG memberships, for use in the EdX discussion forums. This meant that students maintained a consistent group membership both on the EdX platform, and within external activities (for example, students could only see and comment upon lesson designs within their own SIG, resources were mainly shared within SIG, etc.) This in practice creates a number of "parallel courses", each with a common starting point (the videos and prompts), but allowed to develop according to each community's interest.

\subsection{Taking advantage of the crowds}

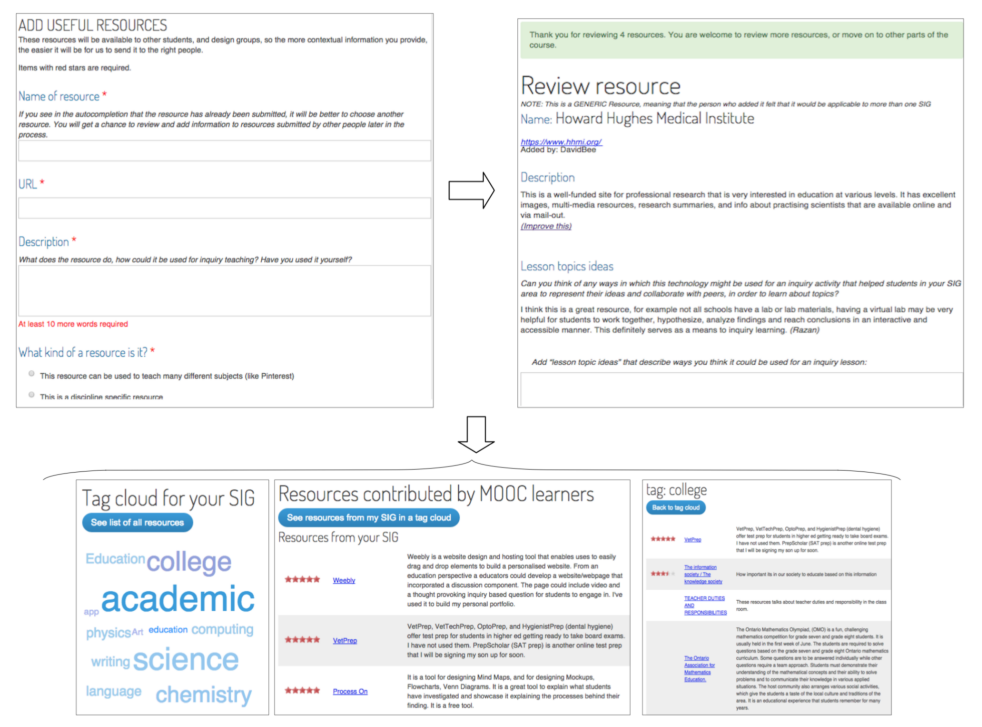

Fig. 1. Resource crowdsourcing script.

Most of the students in the MOOC were in-service teachers, and collectively, they had used and explored hundreds of different digital resources and tools. Some of these resources are specific to their disciplines, such as a simulation of a particular physics phenomenon, whereas others could be used in a wide variety of disciplines in many different ways, such as Google Docs or Socrative. An example of harnessing the collective intelligence of the MOOC participants is the resource brainstorming script, where we asked teachers to share their favorites, with no limit on how many they could contribute. 
First, we asked students to enter digital resources that they had personally found useful, or had heard positive things about. They added title, description, URL, and tags, and chose whether the resource was discipline-specific or "of general use". Once the resources had been submitted, the next step was for students to rate and review resources submitted by others. Upon entering this activity, students were presented with a random resource submitted either by a SIG member, or marked as "for general use" (i.e., applicable to all SIGs). They were allowed to modify the description, add more tags, and comment on how this resource could be used pedagogically, as well as to rate the resource.

Finally, students were able to further explore all the resources submitted, using the meta-data that were attached to the resources. We provided a list of all the resources that were submitted for that SIG (or marked as "applicable to all SIGs"), sorted by score, as well as a tag-cloud where students could choose only the resources belonging to a specific tag (see screenshots in Figure 1).

\subsection{Foundation Strand and Design Strand}

MOOC students vary significantly in their approaches to learning and level of engagement. To address this variation, we needed to make the course appealing and useful to a wide audience, aiming for broad motivation, but also explicitly catering to different groups of learners. Ideally, we would not only offer different ways and levels of participation, but also design the script such that those different groups served as important resources for one another. This is one of the real promises of a knowledge community approach, that it captures and benefits from the diversity within the community, as opposed to conventional lecture-based or didactic pedagogies that typically address the lowest common denominator.

We chose a design path with two distinct modes of engagement, but where the different modes of engagement were inter-dependent, and benefited from one another. The levels of engagement can be described as a series of concentric circles.

None of the external (LTI based) activities were available before students had taken our LTI survey (since we could not access learner information from EdX through the LTI API, we had to ask students directly). Students that signed up for the course in EdX, but never completed the LTI survey were considered as auditors. They had access to all the content hosted on the EdX platform (videos, quizzes, discussion forums and further readings), but did not participate in any of the interactive exercises (whether Foundation Strand or Design Strand), and were thus not eligible to receive Course Certificates.

Any student that managed to make it through the LTI registration were considered as part of the Foundations Strand. Just like the auditors, they had access to the video material and other content, but in addition, they were asked each week to complete individual reflections, participate in forum discussions within their SIGs, self-assess their performance, and participate in various inquiry activities that were designed to promote reflection and learning while also providing inspiration and feedback to those in the Design Strand. 
Students in the Design Strand completed the activities of the Foundation Strand, but in addition they had signed up to work as part of a small team on designing a technology-based inquiry lesson. They had access to the Collaborative Workbench as described below, and received weekly prompts to support their work. They built upon the resources and ideas collected by the Foundation Strand students, and received weekly feedback from the community on their design work.

To emphasize that participation in the Design Strand should be seen as an extra opportunity for rich engagement with other learners in creating a product that could be useful in one's future professional work, we explicitly chose to give no course credit for the Design Strand. Students could earn 100\% marks simply by participating in the Foundation Strand activities (including submitting resources, providing feedback on lesson designs and contributing in other ways).

\subsection{Collaborative Workbench}

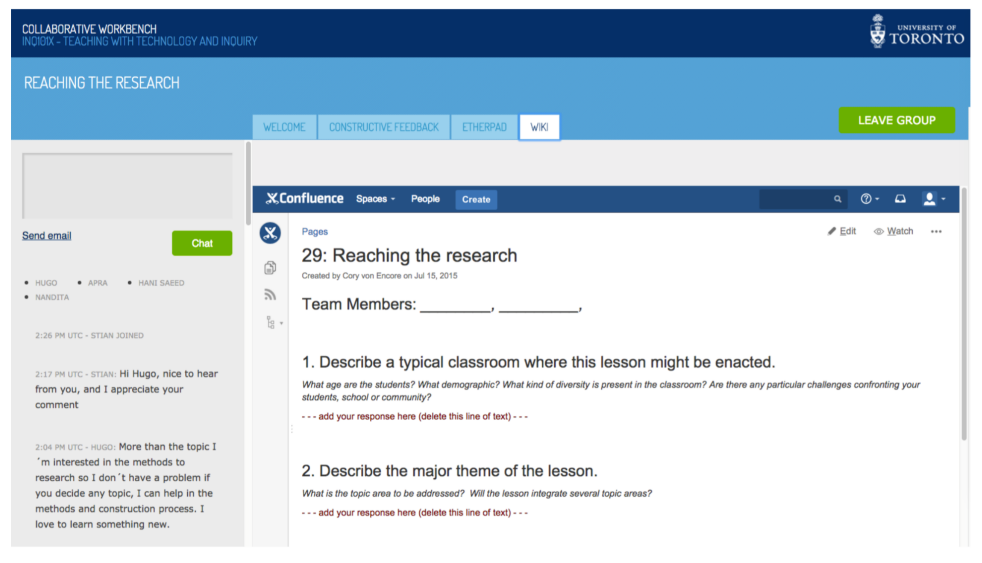

Fig. 2. Collaborative workbench.

Once students had decided on a general topic, and joined together in a team, we needed ways of enabling and sustaining their collaborative effort, creating a sense of a collaborative team, among people who had never met before. A key part of orchestrating the Design strand was the collaborative workbench - -a unified interface that provided a design team with all the necessary information and functionality required for doing its creative work and group coordination. Upon creating a new design team, or joining an existing design team, students entered the full-screen interface shown in Figure 2.

The left sidebar of the Collaborative Workbench contained communication and social presence tools, including a list of the members of the group, with a green symbol showing who was currently online in the collaborative workbench. 
Students could send chat messages, which were persistent (i.e., students could scroll through the entire chat history of the collaborative workbench, including messages that were sent while they were not logged in) - a design that combined the functionality of chat and notice boards. There was also a button to send email, which opened a small window where students could send all group members an e-mail message. This worked by automatically creating an ad-hoc mailing list for the group, maintaining privacy of student e-mail addresses.

The right part of the Collaborative Workbench was for working on content creation and ideation, and had several main "tabs" that allowed various major functions to occur. The first tab contained a welcome message, including prompts and activity tasks for the current week. This message was updated each week. The other tabs varied with each week, often bringing content or ideas from the larger course. For example, the first time that students entered the Workbench, they got access to the resource explorer, to find resources that could inspire their lesson design. A third tab aggregated the feedback and suggestions from other students on their lesson design as it progressed. Because these different tools were presented as tabs instead of separate pages, students could switch between them without loosing context or data. This was important for students who were in the middle of editing the wiki, and wished to look something up in the Etherpad, or in the external review comments.

To begin sketching out ideas and approaches, we embedded Etherpad (live collaborative text editing) as a means of collaboratively developing their design ideas. A new pad was created each week with different prompts. During the first week, students were asked to use the pad to introduce themselves to their group members, and began brainstorming the topic of their lesson, how it was positioned in the wider curriculum, and its length.

A dedicated wiki page for the lesson was introduced in week 2 , to serve as the platform for the final lesson design). This was a Confluence wiki page embedded directly into the workspace, where students were automatically logged in and sent to the right page. This page included a template, with pre-designed headers that would help ensure all major elements were present within their design. Initially, only a few headers were visible, in order to avoid distraction, and keep the design focus on the important early stages (i.e., coming up with a topic, some core technology resources, and a sketch of an activity plan). As weeks went by, new headers were added, corresponding to the weekly theme.

The chat and the Etherpad content were private to the group, and only the lesson overview (title, description, etc) and the wiki text was ever made available to the wider Foundations community. During the final week of the course, we pooled all lesson designs that had been completed, and curated them into a "gallery walk" that was made available to the whole course population This gallery walk was later put on a public URL, and will be available to future course generation, as well as to the general public. 


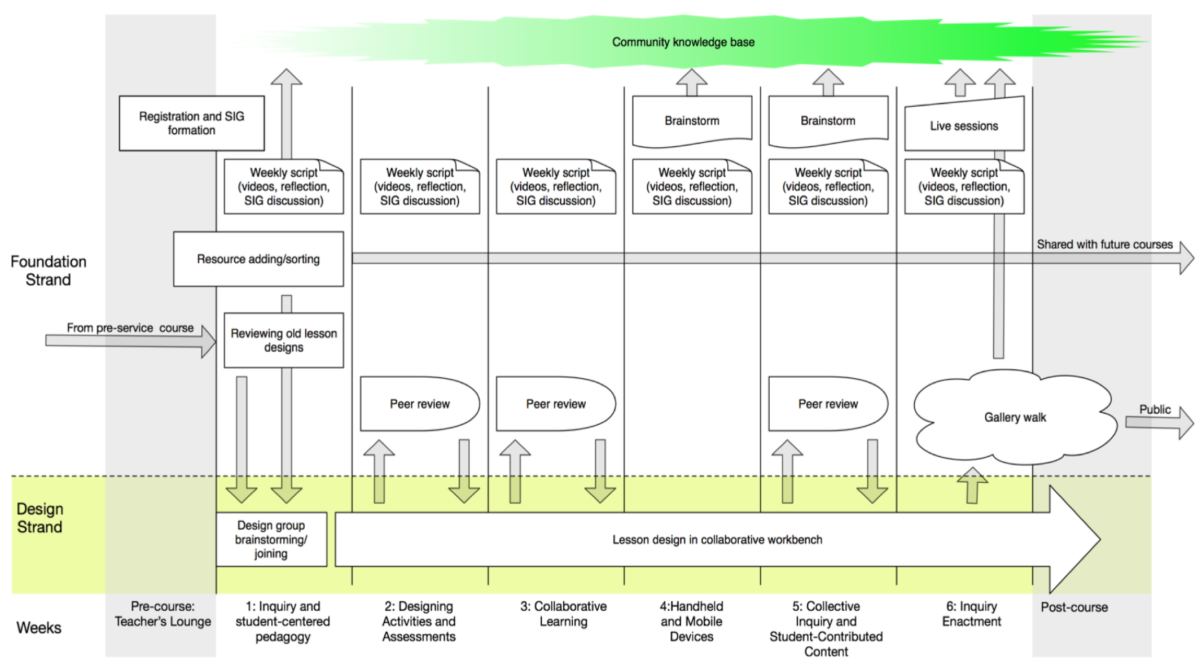

Fig. 3. Graphical depiction of interconnecting MOOC scripts.

\subsection{Scripting across the curriculum}

Designing a complete course according to KCI principles requires a matrix of scripts that fulfill a number of objectives, while responding to course constraints. We had a number of learning objectives for students: we wanted them to learn about and experience a number of different technologies and resources for learning; to become familiar with a number of theoretical approaches and themes and have the opportunity to reflect on how these new ideas relate to their own teaching practices; and to use creativity and critical thinking in integrating the course topics with their existing knowledge to create a lesson design.

We were thus challenged to develop a script that addressed these various learning goals, without overly confusing students. Our design approach took the form of a matrix of scripts. Each week included a pedagogical script where participants worked through the videos, added personal reflections, participated in discussions, and completed short surveys. The Inquiry activities were divided into two categories, the Foundation Strand and the Design Strand. A smaller number of very motivated students could choose to form small persistent teams, which would work on a project to design a complete technology-infused lesson plan, scaffolded with prompts and guidance throughout the entire course. The larger course community would provide input to this process through initial brainstorming, and weekly constructive rounds of peer-review.

Thus, we were able to treat each of the course themes in a rich way, while simultaneously letting a number of course-scripts unfold from week to week. Each week, the students and groups increased in their knowledge and sophistication, having access to a growing personal and community knowledge space. For example, in week three, a student would begin by watching three videos about 
collaborative learning: an academic presentation by Professor Slotta, a school perspective by the UTS Principal, and an example of an application, through an interview with a teacher, and video from an actual classroom.

The student would continue by doing a personal reflection about collaboration (related to their own teaching practice), and respond to several prompts related to collaboration in their discipline-specific SIG (for example, how is collaboration related to physics teaching-for physics teachers). They would then look at the in-progress lesson design by a lesson design group (in their SIG, for example physics teachers trying to teach gravity using augment reality glasses), and add a comment about how this team could incorporate more collaboration into their design. Finally, a student who was a member of a Lesson Design group would log into their Collaborative Workbench, see the weekly prompt (related to collaboration), as well as the peer review comments from all their peers, before they continued work on improving their lesson design document.

Thus, scripts are indexed by the weekly themes, and also have specific interdependencies with each other, including pathways were artefacts are exchanged as a means of connecting micro and macro level scripts (as seen in Figure 3). The graph also shows how the MOOC is connected to previous course offerings through a database of previous lesson designs, how it leaves a legacy for future generations (lesson designs and indexed resource collection), and even how it shares resources with the greater public.

\section{Conclusion}

In this paper, we have described an approach to designing a collaborative curriculum for a MOOC that explicitly addresses learning goals, harnesses the large number of students to crowd-source ideas, groups students based on their interests or characteristics to make the discussion more relevant, enables small intensive teamwork across multiple weeks, and ties together multiple long-lasting collaborative scripts with weekly course topics through a course matrix, and a set of inter-dependencies. We have shown that the KCI framework and a Knowledge Community approach can be applied to a MOOC context, taking advantage of the diverse backgrounds and experiences of MOOC participants, and making the learning more relevant to their professional lives.

In the end, more than 8,000 students registered for the MOOC, of which around 2,200 registered for LTI activities. Students submitted 1,320 resources, with 431 unique tags. 428 students joined one of 142 design groups (Design Strand), and visited the collaborative workbench on average 7 times each during the course, sending an average of 7 chat messages per group. 25 Design Groups were selected as being complete and high quality, and were displayed publicly as part of the gallery walk. While we hope to improve the participation, and especially the completion rate, in future iterations, there was already an impressive amount of knowledge exchange and community building happening in this course. 
A key element in enabling the implementation of this script, was the large amount of external activities that we built. EdX offers no APIs for accessing student information, manipulating cohort membership, reading and responding to forum messages, or otherwise interacting with courses. The LTI protocol is also very minimalistic in the kinds of interactions it enables. However, because all of our LTI services lived on the same server, backed by the same database, we were able to build up a sophisticated student model available to all activities, taking into account group membership, student interests, past activity, etc. By

embedding these LTI activities into the EdX activity progression, instead of offering a completely separate website, students never lost their place, and could easily understand what was expected each week. And hosting activities on our own server, enabled us to generate live dashboards with learning analytics, to be able to respond very rapidly to student behaviour while developing the course.

Unfortunately, the technology developed for this MOOC, while open source and available [4], cannot easily be adapted for other courses. More research and development is required to explore more flexible MOOC platforms or platforms for authoring and running rich collaborative scripts.

\section{References}

1. Downes, S. Connectivism and connective knowledge: essays on meaning and learning networks, 2012.

2. Ferschke, O., Yang, D., Tomar, G., and Rosé, C. P. Positive Impact of Collaborative Chat Participation in an edX MOOC. In Artificial Intelligence in Education, C. Conati, N. Heffernan, A. Mitrovic, and M. F. Verdejo, Eds., vol. 9112. Springer International Publishing, Cham, 2015, 115-124.

3. Fischer, F., and Mandl, H. Knowledge convergence in computer-supported collaborative learning: The role of external representation tools. The Journal of the Learning Sciences 14, 3 (2005), 405-441.

4. Håklev, S. Software for INQ101x. https://github.com/houshuang/survey, 2016.

5. Hickey, D. T., Quick, J. D., and Shen, X. Formative and summative analyses of disciplinary engagement and learning in a big open online course. In Proceedings of the Fifth International Conference on Learning Analytics And Knowledge, LAK '15, ACM (New York, NY, USA, 2015), 310-314.

6. Kulkarni, C. E., Bernstein, M. S., and Klemmer, S. R. Peerstudio: Rapid peer feedback emphasizes revision and improves performance. In Proceedings of the Second (2015) ACM Conference on Learning@ Scale, L@S '15, ACM (New York, NY, USA, 2015), 75-84.

7. McMahon, M. Social constructivism and the world wide web-a paradigm for learning. In ASCILITE conference. Perth, Australia, vol. 327 (1997).

8. Severance, C., Hanss, T., and Hardin, J. Ims learning tools interoperability: Enabling a mash-up approach to teaching and learning tools. Technology, Instruction, Cognition and Learning 7, 3-4 (2010), 245-262.

9. Slotta, J. Knowledge Community and Inquiry. Paper presented and published for the Network of Associated Programs in the Learning Sciences (NAPLES). Tech. rep., 2014.

10. Slotta, J. D., and Najafi, H. Supporting collaborative knowledge construction with web 2.0 technologies. In Emerging Technologies for the Classroom: A Learning Sciences Perspective, N. Lavigne, Ed. 2012. 